\title{
Theoretical Characterization of the Air-Stable, High-Mobility Dinaphtho[2,3-b:2'3'-f]-thiophene Organic Semiconductor
}

\section{Citation}

Sánchez-Carrera, Roel S., Sule Atahan, Joshua Schrier, and Alán Aspuru-Guzik. 2010. Journal of Physical Chemistry 114(5): 2334-2340.

\section{Published Version}

doi:10.1021/jp910102f

\section{Permanent link}

http://nrs.harvard.edu/urn-3:HUL.InstRepos:8364934

\section{Terms of Use}

This article was downloaded from Harvard University's DASH repository, and is made available under the terms and conditions applicable to Open Access Policy Articles, as set forth at http:// nrs.harvard.edu/urn-3:HUL.InstRepos:dash.current.terms-of-use\#OAP

\section{Share Your Story}

The Harvard community has made this article openly available. Please share how this access benefits you. Submit a story. 


\title{
Theoretical Characterization of the Air-stable, High-mobility
}

\section{Dinaphtho[2,3-b:2'3'-f]thieno[3,2-b]-thiophene Organic Semiconductor}

\author{
Roel S. Sánchez-Carrera, ${ }^{\dagger *}$ Sule Atahan-Evrenk, ${ }^{\dagger}{ }^{\dagger *}$ Joshua Schrier, ${ }^{\#}$ \\ and Alán Aspuru-Guzik ${ }^{\dagger^{* *}}$ \\ $\dagger$ Department of Chemistry and Chemical Biology \\ Harvard University \\ Cambridge, Massachusetts 02138 \\ \# Department of Chemistry, \\ Haverford College, \\ Haverford, Pennsylvania 19041
}

*E-mails: rsanchez@fas.harvard.edu; atahan@fas.harvard.edu; aspuru@chemistry.harvard.edu 


\begin{abstract}
Recently, an optimum mobility of $8.3 \mathrm{~cm}^{2} /(\mathrm{Vs})$ has been measured for single-crystal organic field effect transistors based on the dinaphtho[2,3-b: $\left.2^{\prime}, 3^{\prime}-f\right]$ thieno[3,2-b]-thiophene (DNTT) molecule. Here, on the basis of quantum chemistry calculations and molecular dynamics simulations, we investigate the microscopic charge transport parameters of the DNTT molecule and crystal. Our findings confirm that the moderate anisotropy of the hole mobility in DNTT is highly dependent on the presence of in-plane herringbone-like intermolecular interactions with large electronic coupling (transfer integral) values (ca. $0.1 \mathrm{eV}$ ). Also, we demonstrate that the $\pi$ extended heteroaromatic structure leads to strong electronic coupling interactions among neighboring molecules and to a decrease of the intramolecular reorganization energy. In DNTT, thermal modulations of the electronic couplings at $300 \mathrm{~K}$ remain small when compared to those exhibited by the pentacene single-crystal. This theoretical study suggests that heteroacenes are a promising route towards high-mobility organic semiconductor materials.
\end{abstract}




\section{Introduction}

Organic semiconductors are widely investigated as candidates for new generation of thinner, larger, and higher resolution electronic devices. ${ }^{1-4}$ Advances in the scientific understanding and in the device performance of these materials have resulted in organic semiconducting materials that have, in some instances, superseded $\mathrm{Si}$ in performance. ${ }^{5-8}$ Among them, the pentacene molecular crystal, which has emerged as the benchmark of organic molecular semiconductors, has been used to develop organic thin-film transistors with hole mobilities as high as 5.5 $\mathrm{cm}^{2} /(\mathrm{Vs}) .{ }^{9}$ However, building materials with both air stability and high charge mobility has proved to be a very challenging task. ${ }^{1,10-12}$

Recently, a new molecular semiconductor, dinaphtho[2,3-b: $\left.2^{\prime}, 3^{\prime}-f\right]$ thieno[3,2-b]-thiophene (DNTT) was introduced by Takimiya and co-workers ${ }^{13}$ and has gained attention due to its high air-stable mobility values $\left(3.1 \mathrm{~cm}^{2} /(\mathrm{Vs})\right)^{14}$ and its relatively simple synthetic pathway. In addition, a more recent study reported an optimum mobility of $\sim 8.3 \mathrm{~cm}^{2} /(\mathrm{Vs})$ in the saturation regime of single-crystal organic field-effect transistors based on DNTT. ${ }^{15}$ Recent experimental measurements also revealed a moderate anisotropy of the charge mobility of DNTT singlecrystal transistors. ${ }^{16}$ Despite the availability of the experimental evidence of the highperformance of the DNTT molecular crystal, to the best of our knowledge, there is no theoretical characterization that provides a clear explanation for the origin of its high mobility and moderate anisotropic behavior. 
Here, we use quantum-chemical calculations and molecular dynamics simulations to investigate the charge transport characteristics of DNTT in terms of the electronic and vibrational couplings of both the molecule and the crystal. The crystalline structure of DNTT is characterized by the presence of herringbone sheets ( $a b$-plane) with relatively weak coupling between the layers $(c-$ axis). We find that the herringbone packing of DNTT molecules plays a significant role in determining the observed moderate anisotropy of the hole mobilities in DNTT. This is due to the presence of strong electronic couplings between molecular dimers located in the direction of the $a b$-plane. In addition to that, relatively small hole-effective masses are calculated in the direction of the $a$ and $b$ axes. We also find that the microscopic charge transport properties in DNTT are only slightly perturbed by the electron-phonon interactions, unlike in small oligoacene organic semiconductors (i.e., naphthalene and anthracene) or in oligothiophene-based semiconducting materials, where the vibrational couplings play an important role in the description of the transport properties of these materials. ${ }^{17-20}$ Comparison with experiment and pentacene, the wellknown organic semiconductor material, is also highlighted.

\section{Methodology}

The geometry and electronic structure of the isolated DNTT molecule were obtained at the density functional theory (DFT) level by performing geometry optimizations with the Beckethree parameter Lee-Yang-Parr (B3LYP) hybrid density functional ${ }^{21,22}$ and the $6-31 \mathrm{G}(\mathrm{d}, \mathrm{p})$ basis set, ${ }^{23-25}$ as implemented in the Q-Chem software package. ${ }^{26}$ The results of vibrational frequency calculations at the B3LYP/6-31G(d,p) level of theory were then used to calculate the relaxation energy based on the theoretical methodology described by Reimers, ${ }^{27}$ and adapted here for the 
Q-Chem code. The transfer integrals for nearest-neighbor pairs of DNTT molecules were also calculated at the same level of theory.

The electronic-band-structure calculations of the DNTT single-crystal was carried out using DFT with the $\mathrm{PBE}^{28}$ (Perdew-Burke-Ernzerhof) exchange-correlation functional and plane-wave basis set as implemented in the VASP code. ${ }^{29-31}$ Electron-ion interactions were described using the projector augmented wave (PAW) method. ${ }^{32,33}$ The kinetic energy cutoff on the wave function expansion was $300 \mathrm{eV}$ and the self-consistent calculations were carried out with a $14 \times 14 \times 12 \mathrm{k}$ point mesh. The inverse effective mass tensor was calculated using Sperling's centered difference method at the valence band edge of DNTT with $d k=0.02(2 \pi / \AA)$.

To investigate the modulation of the transfer integrals by the thermal motions in the crystal (see electron-vibration coupling section below), we employed a combined approach that uses molecular dynamics (MD) simulations and quantum-chemical calculations. First, a $4 \times 4 \times 4$ supercell containing 128 molecules was created using replicas of the experimentally determined DNTT unit cell. To describe the interatomic potentials in the DNTT crystal, we used the MM3 force field with the addition of electrostatic potential derived charges obtained at the B3LYP/6$31 \mathrm{G}(\mathrm{d}, \mathrm{p})$ level of theory. All bonds to hydrogen atoms were constrained at ideal bond lengths with the help of RATTLE algorithm. ${ }^{34}$ A 250 ps simulation was performed with time steps of 2 fs in NVT ensemble for $\mathrm{T}=300 \mathrm{~K}$ with periodic boundary conditions. Only the last $150 \mathrm{ps}$ of the total simulation time were included in our analysis. The atomic coordinates of the supercell were saved every 30 fs along the trajectory to give 5000 total snapshots. All nuclear dynamics in this work were carried out with TINKER 4.2 molecular modeling package. ${ }^{35-37}$ Finally, the atomic 
coordinates of the three different molecular dimers, illustrated in Figure 1b, were extracted from the MD snapshots and used to calculate transfer integrals at the B3LYP/6-31G(d,p) level of theory. The results of the 15000 ab-initio evaluations of the intermolecular electronic couplings were used to estimate the strength of the nonlocal source of electron-phonon interactions.

The reported crystalline structure of DNTT based on the X-ray structural analysis has almost planar molecules packed along the $a b$-plane in the typical herringbone structure. ${ }^{13}$ The unit cell, which belongs to the $P 2_{1}$ space group, involves two translationally non-equivalent molecules, giving rise to different types of dimer interactions, as illustrated in Figure 1. In this work, we use the previously reported crystalline structure of DNTT and the generalized methodology described above to investigate the electronic properties and the vibrational couplings of this high-performance organic molecular semiconductor. 
(a)

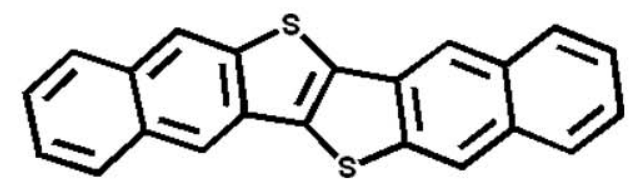

(b)

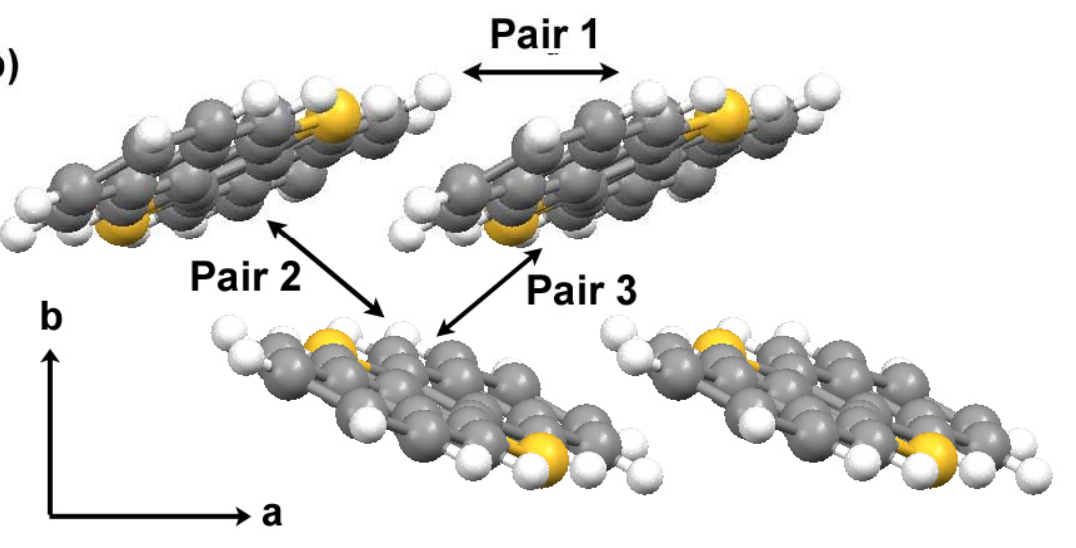

(c)

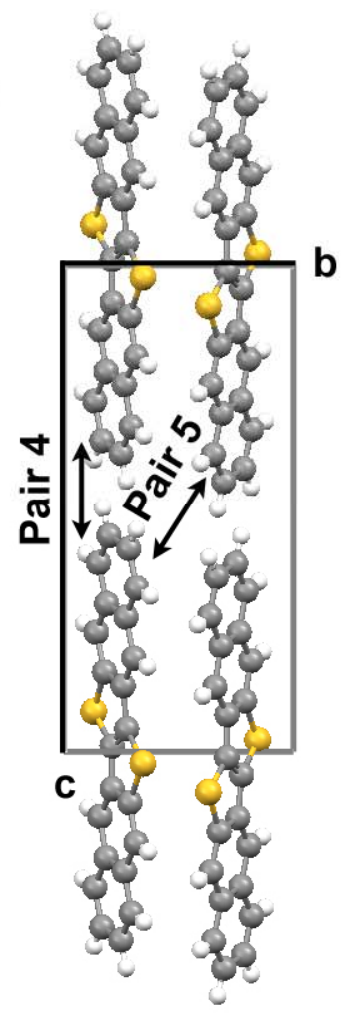

Figure 1. (a) Chemical structure of DNTT. (b) Crystal structure of DNTT in the $a b$-plane. (c) Crystal structure of DNTT in the $b c$-plane $(a=6.187, b=7.662, c=16.21 \AA ; \beta=92.49) .{ }^{13}$ Labels correspond to the DNTT molecular pairs used in the calculation of transfer integrals.

\section{Results and Discussion}

We investigated the microscopic charge transfer characteristics of DNTT in terms of its transfer integrals, electronic band structure, effective masses, and local and nonlocal electron-phonon interactions. Below, we discuss our main findings relevant to the description of the charge transport properties in the DNTT crystal. 
Transfer Integrals and Electronic Band Structure. The electronic properties of single crystals of organic molecules are generally associated with the study of intermolecular transfer integrals $(t)$ and their respective bandwidths $(W)$. Within a simple tight-binding model the valence [conduction] bandwidth results from interaction of the highest occupied molecular orbital (HOMO) [lowest unoccupied molecular orbital (LUMO)] levels of each independent molecule within the crystal. ${ }^{38}$ For instance, in the case of an infinite one-dimensional crystalline stack of molecules the total bandwidth $W$ is equal to $4 t^{39,40}$ In a simpler model, involving only two interacting molecules, the splitting of the HOMO [LUMO] energy levels on the two molecules is equal to $2 t \cdot{ }^{38}$ However, when the two interacting molecules are not symmetrically equivalent, the energy-splitting model leads to inaccurate results due to site energy differences induced by electrostatic effects such as polarization. ${ }^{41}$ Here, we employ a more rigorous computational method, similar to those developed by Norton, et al. ${ }^{42}$ and Senthilkumar, et al. ${ }^{43}$ to estimate transfer integrals between the molecular dimers shown in Figure 1.

The calculated transfer integrals of the selected molecular dimers in the $a b$-plane and along the $c$-axis of the DNTT molecular crystal are given in Table 1. In agreement with recently reported results based on the extended Hückel method, ${ }^{16}$ relatively large values of the transfer integral are found for dimers along the $a$-axis ( $t_{\mathrm{a}}$, pair 1 in Figure 1) and along the diagonal directions in the $a b$-plane ( $t_{\mathrm{d} 1}$ and $t_{\mathrm{d} 2}$, pair 2 and pair 3 in Figure 1, respectively). Interestingly, even though short intermolecular distances are found along the $c$-axis (ranging between 2.5 and $3.5 \AA$ ), only small transfer integral values for holes and electrons are found along this direction (see Table 1). In this view, our calculated electronic couplings support the experimentally observed twodimensional character of the charge transport properties in DNTT. ${ }^{16}$ For comparison, the 
pentacene crystal shows a hole transfer integral value of $85 \mathrm{meV}$ along the diagonal direction comparable to that of DNTT (-94 meV); however, along the $\pi$-stacking direction, the pentacene crystal presents a rather moderate hole transfer integral value of $36 \mathrm{meV}$, whereas the DNTT crystal shows a value, which is twice as large along the same $\pi$-stacking direction (i.e., along the $a$-axis).

Table 1. DFT estimates of the transfer integral for molecular pairs in the DNTT crystal (see Figures 1 for labels).

\begin{tabular}{cccc}
\hline \hline & hole $(\mathrm{meV})$ & electron $(\mathrm{meV})$ & ${\text { hole }(\mathrm{meV})^{\mathrm{a}}}^{2}$ \\
\hline$t_{\mathrm{a}}$, pair 1 & 81 & -27 & 79 \\
$t_{\mathrm{d} 1}$, pair 2 & 29 & 37 & 52 \\
$t_{\mathrm{d} 2}$, pair 3 & -94 & -45 & 86 \\
$t_{\mathrm{c} 1}$, pair 4 & 13 & 0 & - \\
$t_{\mathrm{c} 2}$, pair 5 & 0 & 8 & - \\
\hline
\end{tabular}

[a] $\overline{\text { Calculation based on extended Hückel molecular orbitals from Ref. 16, for comparison. }}$

To gain a better understanding of the strength of the electronic couplings in the $a b$-plane (i.e., the herringbone plane), we have also looked at the details of the three-dimensional electronic band structure in DNTT. The calculated band structure along various high symmetry directions in reciprocal space is shown in Figure 2. Both the valence and conduction bands consist of two subbands arising primarily from the interaction of the HOMO and LUMO levels of the two translationally non-equivalent molecules present in the unit cell. Figure 2 also displays the calculated density of states (DOS), which shows that there are no contributions from other 
molecular states to either the valence or the conduction band. The estimated HOMO and LUMO bandwidths are about $670 \mathrm{meV}$ and $370 \mathrm{meV}$, respectively. In this case, the HOMO bandwidth is slightly larger than that of the pentacene crystal $(600 \mathrm{meV}) ;{ }^{44}$ however, the LUMO bandwidth for the DNTT crystal is almost half of that previously reported for the pentacene crystal. ${ }^{44}$

The observed splitting into two subbands for the valence and conduction band highlights the presence of significant intermolecular interactions not only along the short crystalline axes, but also along the diagonal directions within the herringbone plane. In fact, the largest valence band dispersion occurs along the $\Gamma-\mathrm{X}$ and $\Gamma$-S, corresponding to the $a^{*}$ and $a^{*}+b^{*}$ crystallographic directions, respectively. These results are consistent with the calculated transfer integrals (see Table 1), where significant electronic couplings are found for the $\pi$-stacked dimers (pair 1) and between the diagonal dimers (pair 2 and 3 ). The $\Gamma-Z$ direction corresponds to the $c$-axis and in agreement with the relatively small values of the transfer integrals for holes and electrons $\left(t_{\mathrm{c} 1}\right.$ for holes and $t_{\mathrm{c} 2}$ for electrons $\approx 10 \mathrm{meV}$ ), only small amounts of valence- and conduction-band dispersion occur along this direction. The conduction band shares most of the relevant features of the valence band, with relative dispersion along the $\Gamma-\mathrm{X}, \Gamma-\mathrm{S}$, and $\Gamma-\mathrm{Z}$ directions. However, the main difference between the two transport-related bands occurs along the $\Gamma-Y$ direction ( $b$-axis), along which the valence band has dispersion and the conduction band is completely flat. This difference can be understood by examination of the charge density patterns of the HOMO and LUMO wavefunctions. For example, along the $b$-axis, the electronic interactions occur mainly between the two translationally non-equivalent molecules in the crystal. Since the LUMO wavefunction (see Figure 4) lacks of significant electron density on the sulfur atoms, the effective overlap of the wavefunctions (and consequently the band dispersion) would be, in 
principle, smaller than that of the HOMO. On the other hand, the electron density present on the sulfur atoms of the HOMO level (see Figure 4) promotes effective electronic interactions and significant band dispersion, as observed in Figure 2.

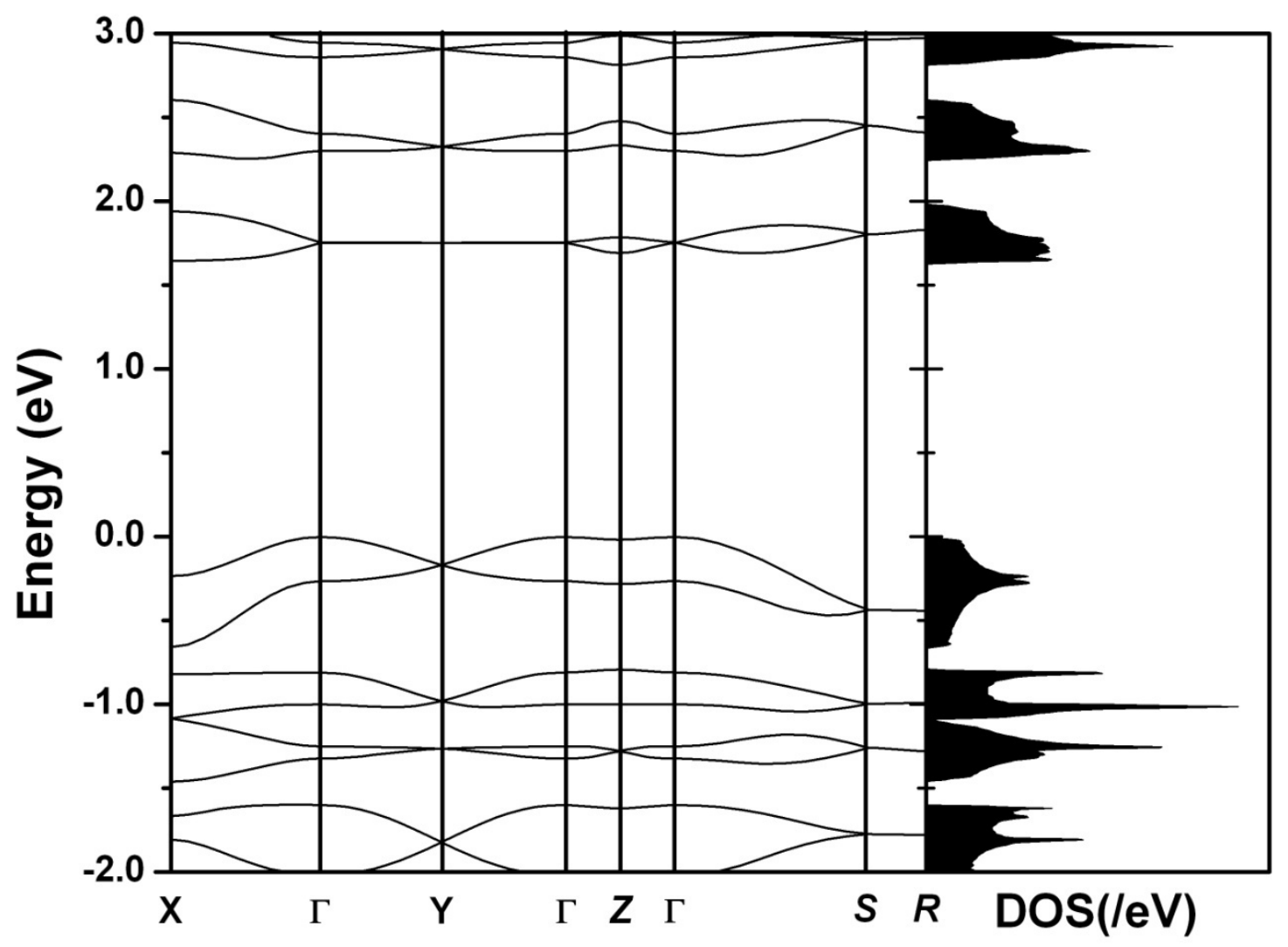

Figure 2. Calculated electronic band structure of the DNTT crystal. The valence band edge is located at the $\Gamma$-point. The right panel illustrates the corresponding density of states. The zero of energy levels corresponds to the valence band edge. The high-symmetry points in units of ( $2 \pi / a$, $2 \pi / b, \quad 2 \pi / c) \quad$ are $\quad \Gamma=(0,0,0), \quad \mathrm{X}=(0.5,0,0), \quad \mathrm{Y}=(0,0.5,0), \quad \mathrm{Z}=(0,0,0.5), \quad \mathrm{S}=(0.5,0.5,0), \quad$ and $\mathrm{R}=(0.5,0.5,0.5)$.

In light of the recent mobility measurements of hole charge carriers in DNTT, ${ }^{15,16}$ we apply the concept of effective mass to describe the charge transport characteristics in this highperformance organic semiconductor. In the case of wide bands, where the thermally populated levels remain close to the valence and conduction band edges, the mobility is governed by the 
electron effective masses that can be computed from the band structure at those band extrema. The tensor for the inverse effective mass $\left(m_{\mathrm{ij}}\right)$ is given by: ${ }^{45}$

$$
m_{i j}^{-1}=\frac{1}{\hbar} \frac{\partial^{2} \varepsilon}{\partial k_{i} \partial k_{j}}
$$

where, subscripts $i$ and $j$ denote the Cartesian coordinates in reciprocal space, $\varepsilon$ the band energy, $\hbar$ the Planck constant, and $k$ the momentum. We have calculated the effective masses at the top of the valence band edge located at the $\Gamma$ point in the DNTT crystal. The effective masses are then analytically derived by fitting Equation 1 to a second-order polynomial; the calculated values are shown in Table 2 .

The lightest hole in DNTT $\left(1.9 m_{0}\right)$ is found along the $a$-axis and a relatively small effective mass is found along the $b$-axis $\left(2.7 m_{0}\right)$. These results are, again, in good agreement with the calculated values of the transfer integrals (see Table 1). For comparison, the average of the hole effective masses for the different polymorphs of the pentacene crystal falls in the range of $1.0 m_{0^{-}}$ $4.0 m_{0}{ }^{44,46-48}$ Within this effective-mass approach, the anisotropy of $m$ implies an anisotropic hole mobility $\mu$ with the highest mobility along the $a$-axis and lower mobility along the $b$-axis, as observed in the experiments. ${ }^{16}$ Quantitatively, we calculate an effective mass ratio $m_{\mathrm{a}} / m_{\mathrm{b}}=0.69$ and in experiments an effective mobility ratio of $\mu_{\mathrm{b}} / \mu_{\mathrm{a}}=0.63$ (one should note that according to the band regime, ${ }^{49}$ the carrier mobility $\mu$ and the effective mass $m$ of the charge carrier are inversely proportional); these ratios are in good agreement. Finally, in Figure 3, we plotted the calculated hole effective masses for all directions within the $a b$-plane. Very interestingly, our results suggest a moderate anisotropy of the hole effective masses in DNTT and contrast with those previously reported for pentacene, in which, accordingly, a very large anisotropy of the hole effective masses within the $a b$-plane has been observed. ${ }^{44}$ 
Table 2. Hole effective mass $m$ (in units of the free electron mass at rest, $m_{0}$ ) calculated at the valence band edge of the DNTT crystal.

\begin{tabular}{ccc}
\hline \hline DNTT & $m / m_{0}$ & parallel to \\
\hline holes at $\Gamma$ & 1.89 & $a+0.050 c$ \\
& 2.73 & $b$ \\
5.28 & $c-0.046 a$ \\
\hline
\end{tabular}

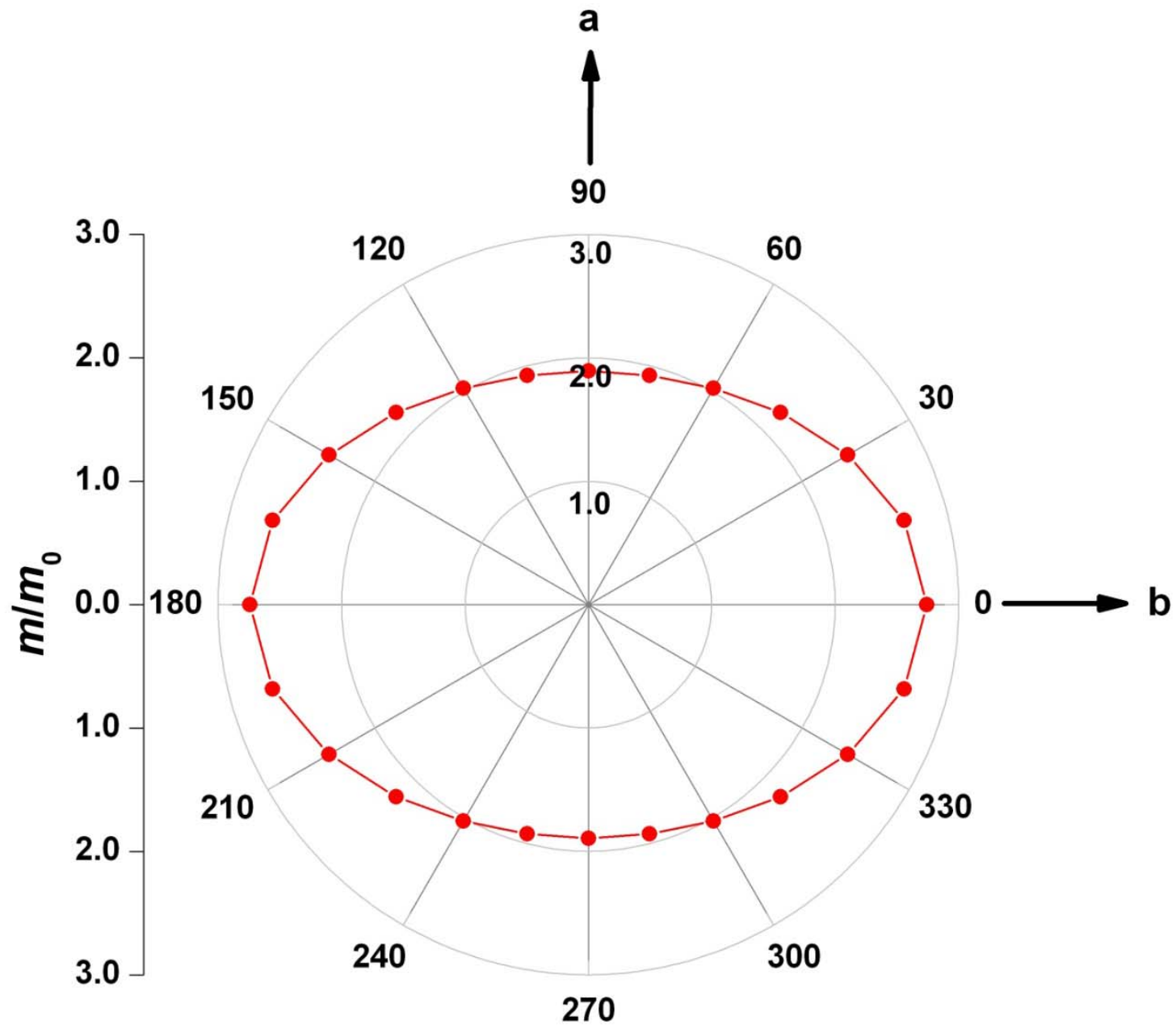

Figure 3. Hole effective masses $m$ (in units of the electron mass at rest, $m_{0}$ ) calculated at the valence band edge of the DNTT crystal as function of the crystallographic direction. 
Electron-Vibration Coupling. In organic molecular crystals, there are two major sources of electron-phonon (vibration) interactions: local and nonlocal couplings. ${ }^{50,51}$ The local electronphonon coupling arises from the modulation of the site energies by intra- and inter-molecular vibrations; its strength is commonly expressed, in the context of electron transfer theory, by the reorganization energy $\lambda_{\text {reorg}}$. Here, we discuss only the intramolecular geometry relaxation contributions to the local coupling, as the polarization of the surrounding molecules (i.e., intermolecular geometry relaxation contributions) are expected to be significantly smaller. ${ }^{52}$ In DNTT the intramolecular reorganization energies associated with the hole- and electronvibrational couplings as calculated from adiabatic potentials are $130 \mathrm{meV}$ and $202 \mathrm{meV}$, for holes and electrons, respectively. In comparison, the corresponding intramolecular $\lambda_{\text {reorg }}$ in pentacene is approximately $97 \mathrm{meV}$ and $132 \mathrm{meV}$, respectively. ${ }^{53,54}$

In Figure 4, we illustrate the contribution of each normal mode to the relaxation energy $\lambda_{\text {rel }}$ (where $\lambda_{\text {rel }} \approx \lambda_{\text {reorg }} / 2$ ) related to the formation of the radical-cation and radical-anion states (only the most strongly coupled modes are shown). The main difference in the $\lambda_{\text {rel }}$ for holes and electrons in DNTT results from a significant drop in the vibronic-coupling interaction with a high-frequency mode at around $1600 \mathrm{~cm}^{-1}$; the magnitude of the coupling with all other modes remains more or less similar in both charged states. The marked decrease in the vibronic coupling interactions in DNTT can be understood in terms of the orbital vibronic coupling constants. ${ }^{55,56}$ According to this model, the vibration coupling constants are large when the molecular deformation along the corresponding normal coordinate considerably distorts the electron density of the related molecular orbitals. In this case, the $1600 \mathrm{~cm}^{-1}$ high energy mode consists of $\mathrm{C}-\mathrm{C}$ stretching within the inner molecular rings. Because part of the electron density 
in the HOMO wavefunction of DNTT is located on the sulfur atoms, the energy of this molecular level is less affected by this vibration than that of the LUMO level, in which the electron density is mainly distributed across the six-aromatic fused rings. Similar arguments describe the presence of a contributing normal mode to the hole-relaxation energy at around $450 \mathrm{~cm}^{-1}$; this vibration involves stretches of the molecule along the short molecular axis via the sulfur atoms and shows no contribution to the electron-relaxation energy. Therefore, the presence/absence of electronic density on the sulfur atoms in the HOMO/LUMO wavefunction of these molecular orbitals provides an explanation for the relevance of this vibration, uniquely to the description of the hole-relaxation energy, as shown in Figure 4.

The decomposition of the intramolecular reorganization energy into individual contributions from the relevant vibrational modes permits to define the contribution of the low-and highfrequency vibrations to the overall value of $\lambda_{\text {reorg. }}$ Here, we looked at contributions of the lowfrequency modes (below $500 \mathrm{~cm}^{-1}$ ) related to hole transfer. We found that, in DNTT, 17\% of the reorganization energy originates from low-frequency vibrations. In pentacene, these contributions account only for $2 \%,{ }^{54}$ and in pentathienoacene, the thiophene equivalent of pentacene, the contribution of the low-frequency vibrations (induced by the sulfur atoms) accounts for as much as $44 \%$ of the calculated intramolecular reorganization energy. ${ }^{57}$ This result points towards the overall superior performance of hybrid thiophene-acene semiconductors, such as DNTT, in which the limited number of sulfur atoms (2 per molecular unit) reduces the contributions of the low-frequency vibrations to the reorganization energy without compromising the environmental stability of the molecule itself (previously, it has been demonstrated that 
functionalization of the molecular backbone with sulfur leads to compounds with an improved environmental stability and modified crystal packing). ${ }^{58,59}$
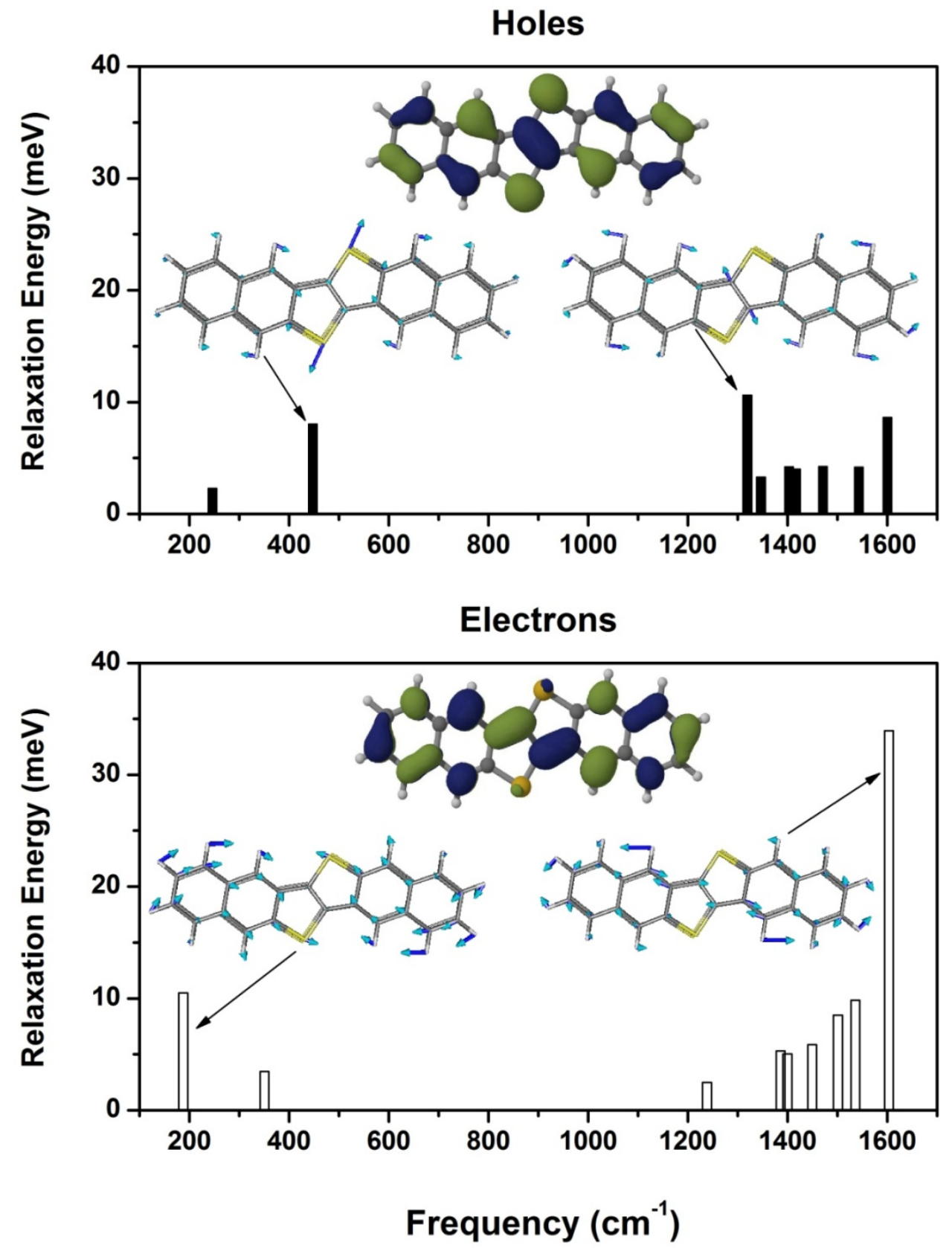

Figure 4. Contributions of the vibrational modes to the hole- and electron relaxation energy in DNTT. The insets show the HOMO and LUMO wavefunctions and the normal modes with strong hole and electron vibronic coupling in DNTT. 
The second source of electron-phonon interactions, namely the nonlocal coupling, arises from the modulation of the transfer integrals by intermolecular vibrations. ${ }^{51}$ Unlike inorganic semiconductors, in an organic semiconductor the molecules are held together by weak forces, therefore the transfer integrals among them are very susceptible to the thermal fluctuations of the molecules within the crystal. To a good approximation, the overall strength of the nonlocal interactions can be obtained by considering the variance of the transfer integrals due to thermal fluctuations. ${ }^{17,60,61}$ To analyze the effect of nonlocal electron-phonon coupling on electronic couplings, we use a combined methodology that uses MD simulations and ab initio quantumchemistry methods. While MD simulations permits access to all (inter- and intra-molecular) vibrational modes in the crystal, $a b$ initio quantum-chemical calculations are used to estimate values of the transfer integrals for selected molecular dimers, whose coordinates are extracted from the MD trajectory at regular time intervals. This combined approach represents an extension of the work of Troisi and co-workers ${ }^{61,62}$ and when applied to organic solids relevant to the field of organic electronics, ${ }^{61,63-65}$ it yields a quasi-Gaussian distribution of the transfer integrals as sampled by classical MD simulations (see Figure 5).

In Figure 5, the distributions of the transfer integrals for holes and electrons for pair 1, 2, and 3 of the DNTT crystal are presented. The standard deviations $\sigma$ of the transfer integrals are also indicated in Figure 5. According to Coropceanu et al., ${ }^{17}$ the standard deviation of the transfer integrals, as derived from MD simulations, can be used to estimate the overall strength of the nonlocal contribution $(L)$ :

$$
L=\sigma^{2} /\left(2 k_{\mathrm{B}} \mathrm{T}\right)
$$


where, $k_{\mathrm{B}}$ is the Boltzmann constant and $T$ the temperature. $L$ has a physical meaning similar to that of the reorganization energy in the case of the local coupling. ${ }^{17}$ Using Equation 2, we estimate the values of $L$ at room temperature for both holes and electrons in the three representative dimers in the $a b$-plane of the DNTT crystal. We find that for most of the selected molecular dimers, $L$ falls in the range of 5-15 meV for both holes and electrons. These values of $L$ are 5 or more times smaller than the calculated transfer integrals obtained at the equilibrium configuration of the MD simulation (see Supporting Information). Thus, the contribution of the nonlocal interactions appears to be too weak to result in any significant vibration-assisted contribution to the charge transport properties in DNTT.

As a comparison, the estimated value of $L$ for holes in DNTT along the diagonal direction (along the so-called herringbone direction) are even smaller than those obtained along a similar orientation in the pentacene crystal (for example, in the case of pentacene, $L=21 \mathrm{meV}$ for herringbone dimers). ${ }^{64}$ In a similar spirit, although a different Hamiltonian was employed to compute the transfer integrals, the recent theoretical investigations of Sleigh et al. ${ }^{66}$ are also in good agreement with the above arguments. For instance, in Ref. 66, the estimated standard deviations of the hole transfer integrals in the pentacene crystal are almost twice as large as the ones calculated for the DNTT crystal. 
HOLES

Pair 1

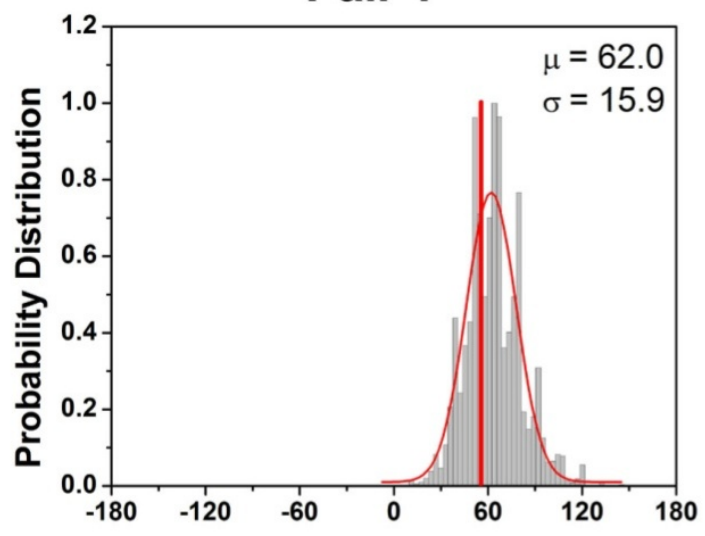

Pair 2

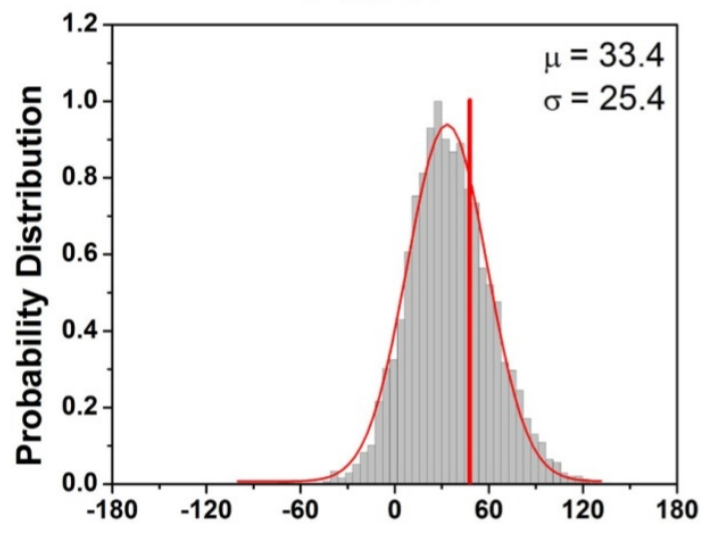

Pair 3

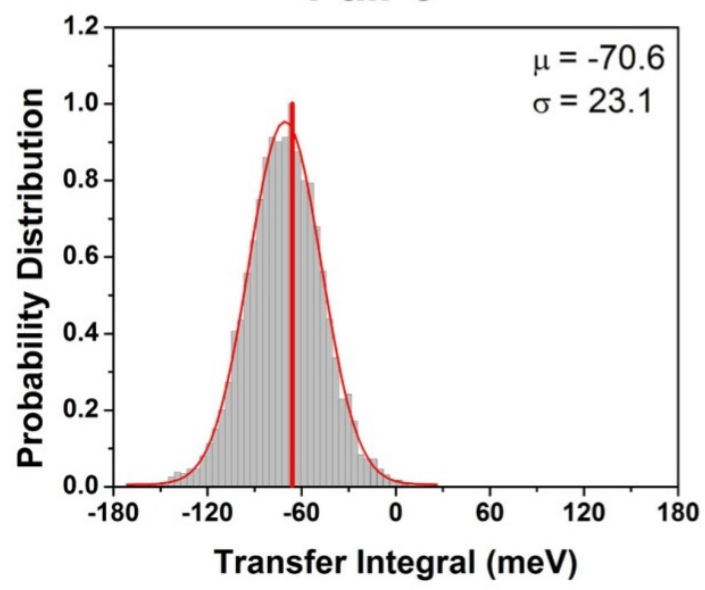

ELECTRONS

Pair 1

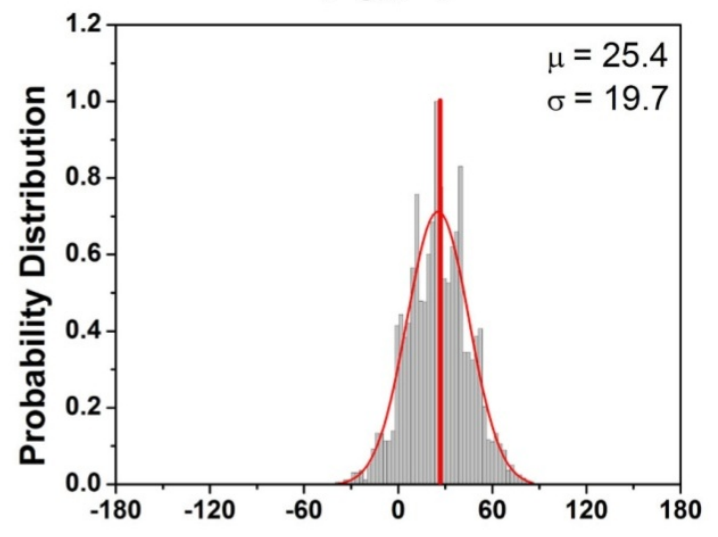

Pair 2

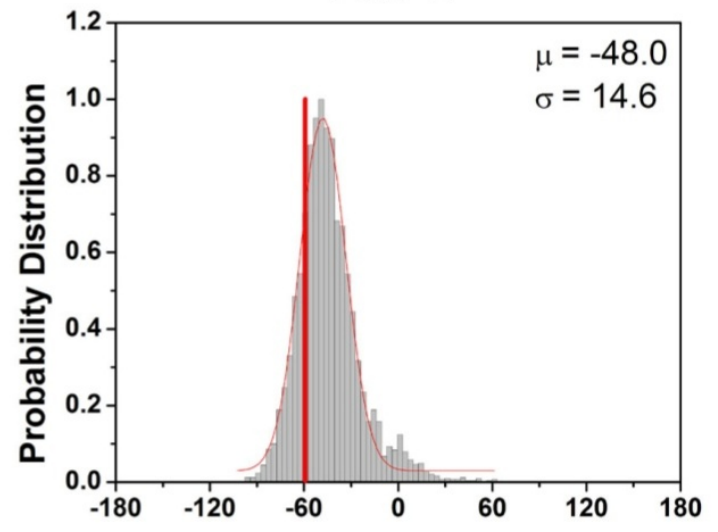

Pair 3

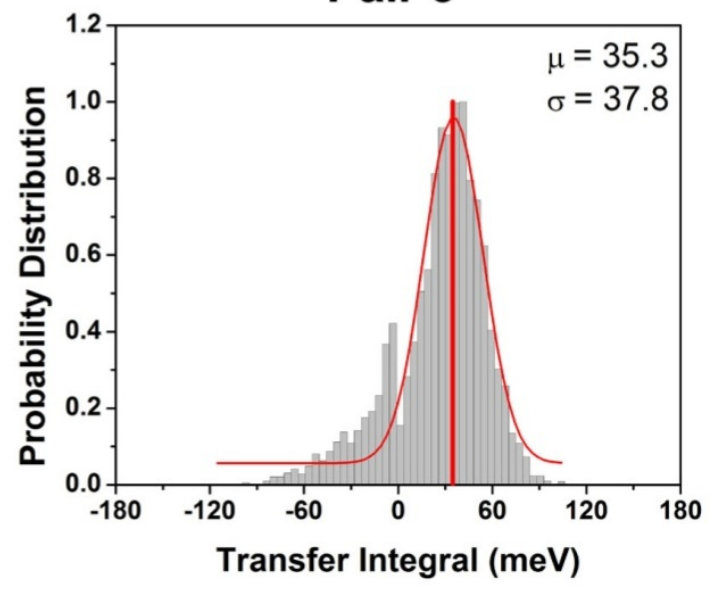

Figure 5. Normalized probability distribution of the transfer integrals for holes and electrons in pair 1, pair 2, and pair 3 of the DNTT crystal. The average value $\mu$ and the standard deviation $\sigma$ are also reported. The single vertical lines correspond to transfer integrals calculated at the equilibrium configuration of the MD simulation. 


\section{Conclusions}

We have investigated the electronic and vibrational couplings in the molecular and crystalline forms of the dinaphtho[2,3-b: $\left.2^{\prime}, 3^{\prime}-f\right]$ thieno[3,2-b]-thiophene molecule. The transfer integral and band structure calculations confirm that the transport of the positive charge carriers is mostly two-dimensional along the $a b$-plane, where herringbone-like intermolecular interactions are dominant. Only slight dispersion and relatively small values of the transfer integrals are found along the $c$-axis, which is almost perpendicular to the herringbone plane. Consistent with recent experimental measurements, calculated effective masses in the $a b$-plane explain the moderate anisotropy observed in the hole mobility of the DNTT crystal. Also, the moderate anisotropy of the calculated hole effective masses in DNTT is in contrast with that of the pentacene crystal, where a large anisotropy of the hole effective masses has been previously reported.

Our results also indicate that the sulfur atoms in DNTT play a dual role in the description of its microscopic charge transport properties because: (1) they promote significant overlap of the molecular orbitals via intermolecular S...S interactions, and (2) although the members of the oligothiophene family of organic semiconductors are characterized by large intramolecular reorganization energies due to low-frequency vibrations induced by the sulfur atoms, the limited presence of sulfur atoms in DNTT (two atoms per molecular unit) accounts for the reduction in the value of the intramolecular reorganization energy. 
Finally, we have investigated the role of the nonlocal electron-phonon couplings in DNTT. Not surprisingly, the $\pi$-extended heteroaromatic molecular structure of DNTT, with its six fused aromatic rings (that is, a relative high molecular-weight-compound) and strong intermolecular electronic couplings, leads to relatively weak nonlocal electron-phonon couplings at room temperature. However, more systematic studies, for example MD simulations at different temperatures, are required to effectively assess the role of the intermolecular vibrations in the DNTT molecular crystal. The results of the present work suggest that the hybridization of different molecular fragments (thiophene and acene) represents a viable route for the design of new organic semiconductors with superior charge transport properties. In this regard, a combinatorial approach for finding novel heteroacene molecular structures is a very promising research direction and further theoretical studies are currently under way.

Acknowledgements. We thank J. Krich, D. Rappoport, A. Perdomo, and L. Vogt for stimulating discussions. R. S. S. thanks the Mary-Fieser Postdoctoral Fellowship at Harvard University. We thank the High Performance Technical Computing Center at the Faculty of Arts and Sciences of Harvard University for invaluable support.

Supporting Information Available: DFT estimates of the transfer integral for molecular pairs in the DNTT calculated at the equilibrium configuration of the MD simulation, DFT estimates of the relaxation energy related to hole and electron transport of DNTT, and DFT/MD estimates of the contributions of the nonlocal hole- and electron-phonon interactions in DNTT. 


\section{References}

(1) Anthony, J. E. Chem. Rev. 2006, 106, 5028-5048.

(2) Bendikov, M.; Wudl, F.; Perepichka, D. F. Chem. Rev. 2004, 104, 4891-4945.

(3) Fichou, D.; Bachet, B.; Demanze, F.; Billy, I.; Horowitz, G.; Garnier, F. Adv. Mater. 1996, $8,500-504$.

(4) Garnier, F.; Horowitz, G.; Peng, X. H.; Fichou, D. Adv. Mater. 1990, 2, 592-594.

(5) Jurchescu, O. D.; Baas, J.; Palstra, T. T. M. Appl. Phys. Lett. 2004, 84, 3061-3063.

(6) Podzorov, V.; Menard, E.; Borissov, A.; Kiryukhin, V.; Rogers, J. A.; Gershenson, M. E. Phys. Rev. Lett. 2004, 93, 086602.

(7) Reese, C.; Chung, W. J.; Ling, M. M.; Roberts, M.; Bao, Z. N. Appl. Phys. Lett. 2006, 89, 202108.

(8) Sundar, V. C.; Zaumseil, J.; Podzorov, V.; Menard, E.; Willett, R. L.; Someya, T.; Gershenson, M. E.; Rogers, J. A. Science 2004, 303, 1644-1646.

(9) Lee, S.; Koo, B.; Shin, J.; Lee, E.; Park, H.; Kim, H. Appl. Phys. Lett. 2006, 88, 162109.

(10) Anthony, J. E. Angew. Chem. Int. Ed. 2008, 47, 452-483.

(11) Chen, M. C.; Kim, C.; Chen, S. Y.; Chiang, Y. J.; Chung, M. C.; Facchetti, A.; Marks, T. J. J. Mater. Chem. 2008, 18, 1029-1036.

(12) Tang, M. L.; Reichardt, A. D.; Miyaki, N.; Stoltenberg, R. M.; Bao, Z. J. Am. Chem. Soc. 2008, 130, 6064-6065.

(13) Yamamoto, T.; Takimiya, K. J. Am. Chem. Soc. 2007, 129, 2224-2225.

(14) Yamamoto, T.; Takimiya, K. J. Photopolym. Sci. Technol. 2007, 20, 57-59.

(15) Haas, S.; Takahashi, Y.; Takimiya, K.; Hasegawa, T. Appl. Phys. Lett. 2009, 95. 
(16) Uno, M.; Tominari, Y.; Yamagishi, M.; Doi, I.; Miyazaki, E.; Takimiya, K.; Takeya, J. Appl. Phys. Lett. 2009, 94, 223308.

(17) Coropceanu, V.; Sánchez-Carrera, R. S.; Paramonov, P.; Day, G. M.; Brédas, J. L. J. Phys. Chem. C 2009, 113, 4679-4686.

(18) da Silva Filho, D. A.; Coropceanu, V.; Fichou, D.; Gruhn, N. E.; Bill, T. G.; Gierschner, J.; Cornil, J.; Brédas, J. L. Philos. Transact. A Math. Phys. Eng. Sci. 2007, 365, 1435-1452.

(19) Grozema, F. C.; van Duijnen, P. T.; Berlin, Y. A.; Ratner, M. A.; Siebbeles, L. D. A. J. Phys. Chem. B 2002, 106, 7791-7795.

(20) Hannewald, K.; Stojanovic, V. M.; Schellekens, J. M. T.; Bobbert, P. A.; Kresse, G.; Hafner, J. Phys. Rev. B 2004, 69, 075211.

(21) Becke, A. D. J. Chem. Phys. 1993, 98, 5648-5652.

(22) Lee, C. T.; Yang, W. T.; Parr, R. G. Phys. Rev. B 1988, 37, 785-789.

(23) Francl, M. M.; Pietro, W. J.; Hehre, W. J.; Binkley, J. S.; Gordon, M. S.; Defrees, D. J.; Pople, J. A. J. Chem. Phys. 1982, 77, 3654-3665.

(24) Harihara.Pc; Pople, J. A. Theor. Chim. Acta 1973, 28, 213-222.

(25) Hehre, W. J.; Ditchfie.R; Pople, J. A. J. Chem. Phys. 1972, 56, 2257-2261.

(26) Shao, Y.; Molnar, L. F.; Jung, Y.; Kussmann, J.; Ochsenfeld, C.; Brown, S. T.; Gilbert, A. T.; Slipchenko, L. V.; Levchenko, S. V.; O'Neill, D. P.; DiStasio, R. A., Jr.; Lochan, R. C.; Wang, T.; Beran, G. J.; Besley, N. A.; Herbert, J. M.; Lin, C. Y.; Van Voorhis, T.; Chien, S. H.; Sodt, A.; Steele, R. P.; Rassolov, V. A.; Maslen, P. E.; Korambath, P. P.; Adamson, R. D.; Austin, B.; Baker, J.; Byrd, E. F.; Dachsel, H.; Doerksen, R. J.; Dreuw, A.; Dunietz, B. D.; Dutoi, A. D.; Furlani, T. R.; Gwaltney, S. R.; Heyden, A.; Hirata, S.; Hsu, C. P.; Kedziora, G.; Khalliulin, R. Z.; Klunzinger, P.; Lee, A. M.; Lee, M. S.; Liang, W.; Lotan, I.; Nair, N.; Peters, 
B.; Proynov, E. I.; Pieniazek, P. A.; Rhee, Y. M.; Ritchie, J.; Rosta, E.; Sherrill, C. D.; Simmonett, A. C.; Subotnik, J. E.; Woodcock, H. L., 3rd; Zhang, W.; Bell, A. T.; Chakraborty, A. K.; Chipman, D. M.; Keil, F. J.; Warshel, A.; Hehre, W. J.; Schaefer, H. F., 3rd; Kong, J.; Krylov, A. I.; Gill, P. M.; Head-Gordon, M. Phys Chem Chem Phys 2006, 8, 3172-91.

(27) Reimers, J. R. J. Chem. Phys. 2001, 115, 9103-9109.

(28) Perdew, J. P.; Burke, K.; Ernzerhof, M. Phys. Rev. Lett. 1996, 77, 3865-3868.

(29) Kresse, G.; Furthmuller, J. Comput. Mater. Sci. 1996, 6, 15-50.

(30) Kresse, G.; Hafner, J. Phys. Rev. B 1993, 47, 558.

(31) Kresse, G.; Hafner, J. Phys. Rev. B 1994, 49, 14251.

(32) Blochl, P. E. Phys. Rev. B 1994, 50, 17953-17979.

(33) Kresse, G.; Joubert, D. Phys. Rev. B 1999, 59, 1758-1775.

(34) Andersen, H. C. J. Comput. Phys. 1983, 52, 24-34.

(35) Dudek, M. J.; Ponder, J. W. J. Comput. Chem. 1995, 16, 791-816.

(36) Kundrot, C. E.; Ponder, J. W.; Richards, F. M. J. Comput. Chem. 1991, 12, 402-409.

(37) Ponder, J. W.; Richards, F. M. J. Comput. Chem. 1987, 8, 1016-1024.

(38) Pope, M.; Swenberg, C. E. Electronic processes in organic crystals and polymers; 2nd ed.; Oxford University Press: New York, 1999.

(39) Haddon, R. C.; Siegrist, T.; Fleming, R. M.; Bridenbaugh, P. M.; Laudise, R. A. J. Mater. Chem. 1995, 5, 1719-1724.

(40) Huang, J. S.; Kertesz, M. J. Chem. Phys. 2005, 122, 234707.

(41) Valeev, E. F.; Coropceanu, V.; da Silva Filho, D. A.; Salman, S.; Brédas, J. L. J. Am. Chem. Soc. 2006, 128, 9882-9886.

(42) Norton, J. E.; Brédas, J. L. J. Chem. Phys. 2008, 128, 034701. 
(43) Senthilkumar, K.; Grozema, F. C.; Bickelhaupt, F. M.; Siebbeles, L. D. A. J. Chem. Phys. 2003, 119, 9809-9817.

(44) de Wijs, G. A.; Mattheus, C. C.; de Groot, R. A.; Palstra, T. T. M. Synth. Met. 2003, 139, $109-114$.

(45) Seeger, K. Semiconductor physics: an introduction; 9th ed.; Springer: Berlin; New York, 2004.

(46) Kitamura, M.; Arakawa, Y. J. Phys. Condens. Matter 2008, 20, 184011.

(47) Troisi, A.; Orlandi, G. J. Phys. Chem. B 2005, 109, 1849-1856.

(48) Yoshida, H.; Sato, N. Phys. Rev. B 2008, 77, 235205.

(49) Sze, S. M. Semiconductor devices, physics and technology; 2nd ed.; Wiley: New York, 2002.

(50) Brédas, J. L.; Beljonne, D.; Coropceanu, V.; Cornil, J. Chem. Rev. 2004, 104, 4971-5003.

(51) Coropceanu, V.; Cornil, J.; da Silva Filho, D. A.; Olivier, Y.; Silbey, R.; Brédas, J. L. Chem. Rev. 2007, 107, 2165-2165.

(52) Norton, J. E.; Brédas, J. L. J. Am. Chem. Soc. 2008, 130, 12377-12384.

(53) Coropceanu, V.; Malagoli, M.; da Silva Filho, D. A.; Gruhn, N. E.; Bill, T. G.; Brédas, J. L. Phys. Rev. Lett. 2002, 89, 275503.

(54) Malagoli, M.; Coropceanu, V.; da Silva Filho, D. A.; Brédas, J. L. J. Chem. Phys. 2004, $120,7490-7496$.

(55) Bersuker, I. B. The Jahn-Teller effect; Cambridge University Press: Cambridge, UK; New York, 2006.

(56) Kato, T.; Yamabe, T. J. Chem. Phys. 2005, 123, 024301. 
(57) Kim, E.-G.; Coropceanu, V.; Gruhn, N. E.; Sánchez-Carrera, R. S.; Snoeberger, R.; Matzger, A. J.; Brédas, J. L. J. Am. Chem. Soc. 2007, 129, 13072-13081.

(58) Laquindanum, J. G.; Katz, H. E.; Lovinger, A. J. J. Am. Chem. Soc. 1998, 120, 664-672.

(59) Wex, B.; Kaafarani, B. R.; Schroeder, R.; Majewski, L. A.; Burckel, P.; Grell, M.; Neckers, D. C. J. Mater. Chem. 2006, 16, 1121-1124.

(60) Gosar, P.; Choi, S. I. Phys. Rev. 1966, 150, 529-538.

(61) Troisi, A.; Orlandi, G. J. Phys. Chem. A 2006, 110, 4065-4070.

(62) Troisi, A.; Orlandi, G. Phys. Rev. Lett. 2006, 96, 086601.

(63) Martinelli, N. G.; Olivier, Y.; Athanasopoulos, S.; Ruiz-Delgado, M. C.; Pigg, K. R.; da Silva, D. A.; Sánchez-Carrera, R. S.; Venuti, E.; Della Valle, R. G.; Brédas, J. L.; Beljonne, D.; Cornil, J. Chemphyschem 2009, 10, 2265-2273.

(64) Ruiz-Delgado, M. C.; Pigg, K. R.; da Silva Filho, D. A.; Gruhn, N. E.; Sakamoto, Y.; Suzuki, T.; Osuna, R. M.; Casado, J.; Hernández, V.; Navarrete, J. T. L.; Martinelli, N. G.; Cornil, J.; Sánchez-Carrera, R. S.; Coropceanu, V.; Brédas, J. L. J. Am. Chem. Soc. 2009, 131, $1502-1512$.

(65) Troisi, A. Adv. Mater. 2007, 19, 2000-2004.

(66) Sleigh, J. P.; McMahon, D. P.; Troisi, A. Appl. Phys. A: Mater. Sci. Process. 2009, 95, $147-152$. 